correlations were shown between Secure and Insecure attachment, the attachment variables Coherence of Mind, Coherence of Transcript and Full Scale Intelligence Quotient. No statistically significant correlations were obtained in the FT-group.

Conclusions Extremely Preterm born, when young adults, shows significantly lower IQ-scores, have negative self and positive others model and shows a higher proportion of insecure attachment. To our knowledge, this is the first study to report data on EPT and its impact on the attachment organization in adulthood. Insecure attachment, low IO and prematurity may be considered as significant risk factors for developing psychopathology, they deserve careful attention in future research and clinical follow-ups.

\section{SOCIODEMOGRAPHIC AND NEONATAL FACTORS ASSOCIATED WITH EARLY CHILDHOOD SOCIAL- COMMUNICATION DIFFICULTIES IN CHILDREN BORN PRETERM}

doi:10.1136/archdischild-2012-302724.1224

${ }^{1} \mathrm{HS}$ Wong, ${ }^{2} \mathrm{~A}$ Huertas-Ceballos, ${ }^{1} \mathrm{FM}$ Cowan, ${ }^{1} \mathrm{~N}$ Modi, Medicines for Neonates Investigator Group. ' $S e c t i o n$ of Neonatal Medicine, Imperial College London; ${ }^{2}$ Neonatal Intensive Care Unit, University College London Hospital, London, UK

Background The Quantitative Checklist for Autism in Toddlers (Q-CHAT) is a parent-completed questionnaire providing a quantitative measure of early childhood social-communication difficulty (Allison et al, J Autism Dev Disord, 2008). The Q-CHAT scores of children born preterm are higher than the general population, indicating greater autistic traits (Wong et al, Neonatal Society Proceedings 2012 Spring Meeting).

Aim To examine sociodemographic and neonatal factors associated with social-communication abilities in preterm infants at 24 months corrected age.

Methods The parents of children born at $<30$ weeks gestation and enrolled in a study evaluating routinely collected neurodevelopmental data were asked to complete the Q-CHAT. Children with severe neurosensory disabilities and cerebral palsy were excluded. The effect of factors identified a priori (maternal age, gestation, birthweight z-score, gender, multiple pregnancy, length of mechanical ventilation, supplemental oxygen requirement at 36 weeks postmenstrual age (BPD) and index of multiple deprivation (IMD)) on Q-CHAT scores were examined using univariable and multivariable linear regressions.

Results The Q-CHAT was completed by the parents of 104 children (mean[SD] gestation 27.0[1.7] weeks, when the children were at a mean corrected age of 24.7[2.7] months). On univariable analysis, gestation, multiple pregnancy, BPD and IMD were positively associated with $\mathrm{Q}$-CHAT scores. Low gestation $(p=0.02)$ and higher IMD $(p<0.01)$ were independently associated with higher Q-CHAT scores on multivariable analysis.

Conclusion Preterm birth is a recognised risk factor for autism spectrum disorder. We report a novel finding of high deprivation as an independent predictor of early childhood social-communication difficulty in the preterm population.

\section{PRETERM BORN PRESCHOOLERS' DISABILITIES IN DAILY ACTIVITIES}

doi:10.1136/archdischild-2012-302724.1225

${ }^{1} \mathrm{G}$ Verkerk, 'M Jeukens-Visser, ${ }^{2} \mathrm{~A}$ van Wassenaer-Leemhuis, ${ }^{1} \mathrm{~K}$ Koldewijn, ${ }^{2} \mathrm{~J}$ Kok, IF Nollet. 'Rehabilitation; ${ }^{2}$ Neonatology, Academic Medical Center, University of Amsterdam, Amsterdam, The Netherlands

Aims Do very low birth weight (VLBW) preschoolers without Cerebral Palsy $(\mathrm{CP})$ have disabilities in daily activities and what are risk factors for these disabilities?
Methods Disability in daily activities was assessed with the Dutch Pediatric Evaluation of Disability Inventory (PEDI-NL) in 143 VLBW children, at 44 months corrected age (CA). Children with CP are known to have disabilities and were therefore excluded. Multiple logistic regression analyses were performed to determine the risk factors for disabilities in daily activities. Perinatal and sociodemographic factors, a low (< 1SD) Psychomotor- Developmental Index (PDI) and low (<1SD) Mental Developmental Index (MDI) of the Bayley Scales of Infant Development (BSID II) at 24 months CA were considered as potential risk factors and included in the analyses.

Results One or more disabilities were found in 27 VLBW children (19\%). The highest frequencies were found in mobility (19 (13\%) children) and in social functioning (12 (8\%) children). Logistic regression analyses detected a low $\mathrm{PDI}$ and a low $\mathrm{MDI}$ as risk factors for disability in mobility; R-square 0.211 . For disability in social functioning, a low MDI and being first born were detected as risk factors: R-square 285.

Conclusions At school entry, one in five VLBW children does have a disability in daily activities especially in mobility and social functioning which may reduce participation with their peers. However, prediction of the disabilities by risk factors is limited. Therefore, adding the PEDI to follow up assessments may enable adequate referral for intervention focussing on participation.

\section{VISUAL SEARCH AND ATTENTION IN VERY LOW BIRTHWEIGHT (VLBW) PRESCHOOLERS}

doi:10.1136/archdischild-2012-302724.1226

${ }^{1,2} \mathrm{CJA}$ Geldof, ${ }^{3} \mathrm{AG}$ van Wassenaer, ${ }^{3} \mathrm{JH}$ Kok, ${ }^{1} \mathrm{~J}$ Oosterlaan. ${ }^{1}$ Department of Clinical Neuropsychology, VU University Amsterdam; ${ }^{2}$ Rehabilitation and Advice, Royal Dutch Visio; ${ }^{3}$ Department of Neonatology, Emma's Children Hospital, Academic Medical Center, Amsterdam, The Netherlands

Background and Aim Very Low Birth Weight (VLBW) is associated with visual perceptual and visuomotor problems (Geldof et al., 2012). This study investigated the nature of the visual search problems in VLBW children and sought to test the hypothesis that visual search problems originate from deficits in attentional networks.

Methods Visual search and attentional network function was assessed in 105 VLBW children and 64 age matched term controls. Visual search performance was investigated with a newly developed paradigm that manipulated target density and ordering of targets. Attentional network function was measured using the Posner Attentional Network Test (ANT; Posner, 2007).

Results Visual search was less efficient in VLBW children compared to controls $\left(\mathrm{F}_{1,167}=4.0 ; p=0.05\right.$ partial $\left.\eta^{2}=0.02\right)$. In addition, VLBW children demonstrated poor executive attention as indicated by lower accuracy levels on the executive attention measure of the ANT $\left(p<0.001\right.$; partial $\left.\eta^{2}=0.08\right)$, but not on the alerting $(p=0.45$; partial $\left.\eta^{2}=0.003\right)$ and orienting $\left(p=0.32\right.$; partial $\left.\eta^{2}=0.01\right)$ attention measures. None of the attention measures significantly predicted visual search efficiency (alerting: $\beta=24 ; p=0.22$; orienting: $\beta=-0.11 ; p=0.65$; executive attention: $\beta=0.17 ; p=0.14$ ).

Discussion VLBW children were characterized by less efficient visual search ability and reduced executive attention. Deficits in executive attention did not explain the deficits in visual search, suggesting that both deficits occur independently of each other.

\section{LONGTERM FOLLOW UP OF COGNITIVE FUNCTION CHILDREN BORN AT THE LIMIT OF VIABILITY AFTER ACTIVE PERINATAL CARE, UMEÅ-UPPSALA STUDY}

doi:10.1136/archdischild-2012-302724.1227

J Karlsson, B Hagglof, A Farooqi. Department of Clinical Sciences, Umea University, Umea, Sweden 
Objective To determine the nature of cognitive function, frequency and severity of cognitive deficit, in 10 to 15-year-old extremely preterm (EPT) children born at 2 tertiary care centers in Sweden adhering to a policy of universal resuscitation of all infants born alive.

Methods The outcomes of 121 surviving EPT children, born 1992-1998, were compared to a control group of 100 term children. Children were assessed with WISC- III at 9-16 years of age. Relationship of perinatal risk factors (PNRF) with the outcomes was examined with multivariate regression analyses (MRA).

Results The EPT group had a mean IQ of 80 (controls 103). 29\% of the EPT children had an IQ below 70 (controls 3.4\%), of which $9 \%$ were under IQ 55 (controls 0 ). Another $30 \%$ of EPT children had IO within the mildly delayed range, 70-84 (controls 12.6\%). The preterm group has a greater variance between cognitive domains, with the language skills as relative strength and spatial thinking as pronounced weakness. MRA revealed that only gestaional age was inversely related to FSIO (B 7.1, P 022) and Performance IQ (B 9.8, $\mathrm{P}<001)$

Conclusion Cognitive dysfunctions remain high in EPT children. These numbers are similar to those from centres with less active perinatal care policies. Very few have severe cognitive impairment that curtail their activities in daily life.

\section{EXECUTIVE FUNCTIONS AT 10-15 YEARS IN CHILDREN BORN AT < 26 WEEKS' GESTATION AFTER ACTIVE PERINATAL CARE: UPPSALA-UMEÅ STUDY}

doi:10.1136/archdischild-2012-302724.1228

J Karlsson, B Hagglof, A Farooqi. Department of Clinical Sciences, Umea University, Umea, Sweden

Aim To determine the nature, frequency and severity of executive dysfunction (EDF) in 10 to 15 -year-old extremely preterm (EPT) children born at 2 tertiary care centers in Sweden adhering to a policy of universal resuscitation of all infants born alive.

Design/methods The outcomes of 121 surviving EPT (86\% of all EPT survivors) children born 1992 through 1998 were compared to a matched control of children born at term. Cognitive function was assessed by WISC-III. Executive functions were assessed by DKEFS Tower of London and ADHD-symptoms by Conners 10-item rating scale. Relationship of perinatal risk factors (PNRF) with the outcomes was examined with appropriate statistical analyses.

Results The EPT children exhibited significant EDF compared with their controls in many EFs such as impulsivity, inattention and planning ability even after controlling for intelligence (IQ). Mean scores in EPT and controls were, respectively, 79 vs 101 in full scale IO, $\mathrm{P}<0.001 ; 2.5$ vs $0.5, \mathrm{P}<0.001$ in Impulsivity; 9.1 vs $11.8, \mathrm{P}<0.001$ in planning ability; and 4.4 vs 1.62 in ADHD symptoms. Severe impairments were exhibited in only a small minority of EPT children. No statistical conclusions were altered when children with substantial neurosensory impairment were excluded. Relation of PNRF will also be discussed.

Conclusions School-aged children who were born EPT in the 1990s are at greater risk for developing EDF and these contribute excess morbidity over cognitive impairment in EPT children. Thus these children require ongoing neuropsychological review throughout their childhood and adolescence.

\section{COMPUTERIZED WORKING MEMORY TRAINING IS EFFECTIVE IN PRETERM BORN CHILDREN AT PRESCHOOL AGE}

doi:10.1136/archdischild-2012-302724.1229

1,2K Hermansen Grunewaldt, ${ }^{1,2} \mathrm{~J}$ Skranes, ${ }^{1,2} \mathrm{AM}$ Brubakk, ${ }^{1,3} \mathrm{GCC}$ Løhaugen. ${ }^{1} \mathrm{Dept}$ of Lab. Medicine, Children's and Women's Health, Norwegian University of Science and Technology; ${ }^{2}$ Dept of Pediatrics, St. Olav University Hospital, Trondheim; ${ }^{3}$ Dept of Pediatrics, Sørlandet Hospital, Arendal, Norway

Background and Aims Working memory (WM) is defined as the skill to retain and manipulate information "on-line" over short periods of time. Deficits in WM are frequently reported in preterm children and are associated with cognitive, behavioural and academic problems. We wanted to evaluate if a software-based computer program would improve WM, attention and behaviour in VLBW children at preschool age.

Methods This prospective study included 20 preterm children. Mean birth weight $1099 \mathrm{~g}(\mathrm{SD}$ 311), mean gestational age 29 weeks(SD 2.8). At age 5-6, the children trained with the Cogmed JM computer program for 10-15 minutes each day, 5 days a week for 5 weeks. The children were assessed before and 4 weeks after training. Effect on trained WM tasks were assessed by improvement measures included in the computer program. Non-trained WM and generalization effects were assessed by neuropsychological (NEPSY) tests. Parental questionnaires regarding ADHD symptoms were performed before and after completed training.

Results The children improved on trained WM tasks (Start-Index: mean 42.1; SD 6.3, Max-Index 60.6; SD 5.7. p $\leq 0.001$.). The group also improved on non-trained WM tasks and showed a generalization effect on auditory attention, phonological awareness and visual and verbal memory. A Stepped-Wedge-Design showed that improvements in test results after training were not due to test-retest effects. There was a trend towards significantly reduced ADHD scores after training.

Conclusion Computerized WM training in VLBW pre-schoolers have positive effects on trained and non-trained WM tasks as well as generalizing effect on verbal and visual learning and memory functions.

\section{THE PREDICTIVE VALUE OF EARLY AEEG PATTERNS FOR FUNCTIONAL OUTCOME AT SCHOOL AGE IN PRETERM INFANTS}

doi:10.1136/archdischild-2012-302724.1230

N Brandenbarg, A Roescher, K van Braeckel, $\mathrm{H}$ ter Horst, A Bos. Department of Pediatrics, Division of Neonatology, University of Groningen, University Medical Center Groningen, Groningen, The Netherlands

Introduction Brain activity of preterm infants can be assessed by amplitude integrated electroencephalography (aEEG).

Objective To determine whether early aEEGs of preterm infants are associated with functional outcome at 7 years.

Methods Eighteen infants participating in a prospective observational study in 2004 were seen for follow-up at median age of 7 years, 5 months. Median GA was 28.9wks (26.7-32.9). aEEGs of $2 \mathrm{~h}$ duration were recorded within $48 \mathrm{~h}$ and one and two weeks after birth. aEEGs were assessed by pattern recognition and by calculating the mean of aEEG amplitude centiles. Functional outcome was determined by assessing intelligence, attention, verbal memory, visuospatial processing, executive functioning and motor skills.

Results In case of burst suppression $<48 \mathrm{~h}$ Total IO was 13 points lower $(P=0.018)$, Performance IQ was 18 points lower $(P=0.016)$ and visuospatial processing scores were $0.68 \mathrm{SD}$ lower $(P=0.027)$. Mean p5 and p50 centiles recorded $<48 \mathrm{~h}$ after birth correlated positively with Total IQ $(r=0.507, r=0.495$, repectively; $P<0.05)$, Performance IQ $(r=0.578, r=0.514$, respectively; $P<0.05)$, selective attention ( $r=0.586, r=0.577$, respectively; $P<0.05$ ), visuospatial processing $(r=0.488, r=0.534$, respectively; $P<0.05)$ and fine motor skills $(r=0.558, r=0.556$, respectively; $P<0.05)$. All correlations remained significant at one week and after adjustment for GA, except for visuospatial processing and fine motor skills.

Conclusion Burst suppression and lower aEEG amplitude centiles within $48 \mathrm{hrs}$ and at one week after birth were associated with 